\title{
Differential effects of cholesterol and budesonide on biophysical properties of clinical surfactant
}

\author{
Hong Zhang ${ }^{1,2}$, Yi E. Wang ${ }^{1}$, Charles R. Neal ${ }^{3}$ and Yi Y. Zuo ${ }^{1}$
}

INTRODUCTION: Corticosteroids have been widely used in clinical medicine as a first-line therapy to modify the inflammatory response in many pulmonary and systemic diseases. Inhaled and intratracheally administered corticosteroids have a particular interest in that their use allows the clinician to circumvent systemic steroid side effects. However, it is vital that corticosteroids delivered via the lungs not interfere with surface activity of the pulmonary surfactant lining layer.

RESULTS: We found differential effects of cholesterol and budesonide on the biophysical properties of a cholesterolfree clinical surfactant preparation, Curosurf. At a low concentration up to $1 \%$, both steroids play a similar role of fluidizing the surfactant film. However, when steroid concentration is increased to $10 \%$, cholesterol induces a unique phase transition that abolishes the surface activity of the Curosurf film. By contrast, $10 \%$ budesonide simply fluidizes the film, thus having only limited effects on surface activity.

DISCUSSION: Together with those of a previous study using a cholesterol-containing surfactant, our findings suggest that cholesterol-free surfactant preparations may be more advantageous than cholesterol-containing preparations as a carrier of budesonide because a larger amount of the drug may be delivered to the lungs without significantly compromising the surface activity of pulmonary surfactant.

METHODS: Langmuir balance was used to study the effect of cholesterol and budesonide added at different concentrations on surface activity of Curosurf. Atomic force microscopy (AFM) was used to reveal their effects on the interfacial molecular organization and lateral structure of Curosurf films.

C orticosteroids, because of their anti-inflammatory actions, have commonly been used to modify the course of chronic lung disease in ventilated premature infants $(1,2)$. However, the use of systemic corticosteroids all but ceased (3) after concern was raised regarding its adverse effects on the developing brain (4). In efforts to overcome the adverse effects of systemic corticosteroids, significant attention has been given to topical administration of corticosteroids directly to the lungs. Two different pulmonary drug-delivery methods have been clinically tested: inhalation delivery of steroid aerosols (5-7) and, very recently, intratracheal instillation of steroids using exogenous surfactant as a carrier (8-11).

Despite its low risk-benefit ratio, pulmonary delivery of corticosteroids requires a comprehensive understanding of the molecular interaction between the corticosteroids and the pulmonary surfactant. Pulmonary surfactant is synthesized by alveolar type II epithelial cells, and it coats the entire inner surface of the respiratory tract as a thin film (12). It consists of $\sim 80 \mathrm{wt} \%$ phospholipids, $5-10 \%$ neutral lipids (primarily cholesterol), and $5-10 \%$ proteins (13). The main function of this surfactant film is to reduce the alveolar surface tension, thus maintaining a large surface area of the lungs for effective gas exchange (12). Therefore, it is critical that corticosteroids delivered via the respiratory system not impair the biophysical properties of the pulmonary surfactant.

All corticosteroids are biochemically derived from cholesterol and hence share a close structural similarity with cholesterol (14). Figure 1 compares the molecular structure of cholesterol and a commonly used inhaled corticosteroid, budesonide. Both molecules feature three cyclohexanes and one cyclopentane in a fused ring structure, with different functional groups attached to this basic skeleton. Recent basic research found that cholesterol plays a vital role in regulating the phospholipid phase behavior of surfactant films and membranes (15-17). Depending on its concentration, cholesterol can significantly alter the molecular organization of surfactant films, thus varying their biophysical properties. In particular, cholesterol at a supraphysiological concentration can inhibit the surface-tension-lowering ability of exogenous surfactants $(16,17)$. These fundamental studies with cholesterol raise a caveat regarding the use of inhaled corticosteroids, related to a similar effect in inhibiting pulmonary surfactant.

As compared with research on cholesterol, there have been very few in vitro studies on the interaction between pulmonary surfactant and corticosteroids (8,18-20). Palmer et al. evaluated the effect of budesonide on an exogenous surfactant (bovine lipid extract surfactant, BLES) using a captive bubble surfactometer (18). It was found that budesonide added at a clinically relevant concentration ( $\sim 0.6 \mathrm{wt} \%$ of surfactant) impaired the surface-tension-lowering ability of BLES. By contrast, using a 

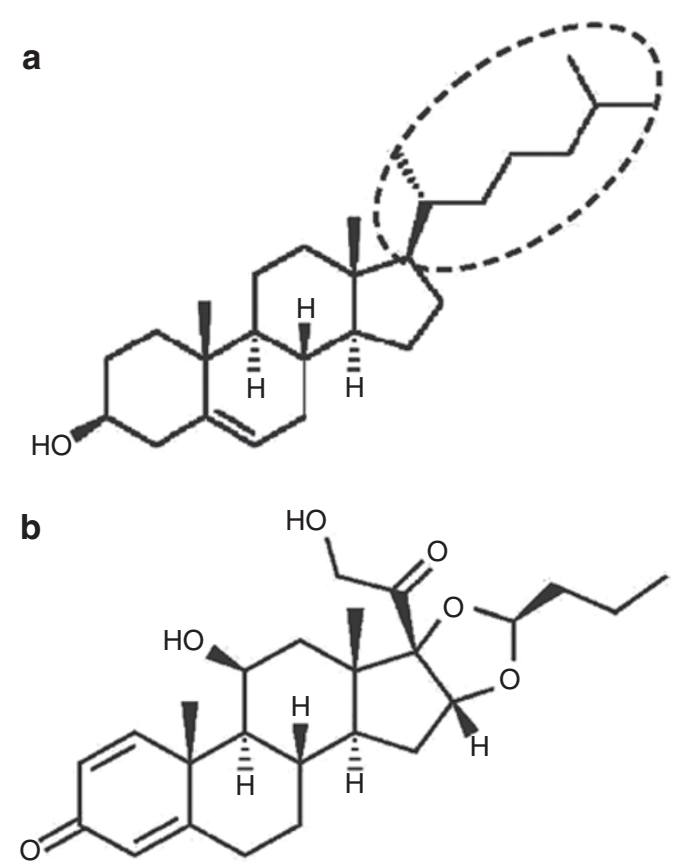

Figure 1. Chemical structures of (a) cholesterol and (b) budesonide. The unique alkyl side chain at the $\mathrm{C}-17$ position of the cholesterol molecule is depicted in panel a.

pulsating bubble surfactometer, Yeh et al. showed that $2 \%$ budesonide added to a different exogenous surfactant (Survanta) did not interfere with its surface activity (8).

Given the conflicting results in the literature, and the importance of developing inhaled/instilled corticosteroid therapy for treating chronic lung disease, we compared the effect of cholesterol and budesonide on the surface activity and molecular organization of a cholesterol-free clinical surfactant preparation, Curosurf, using the combination of Langmuir balance and atomic force microscopy (AFM). Curosurf has been studied as a carrier for intratracheal administration of corticosteroid in a preterm lamb model $(9,11)$. Langmuir balance was used to study the effect of cholesterol and budesonide added at different concentrations on surface activity (i.e., the surface-tensionlowing ability) of Curosurf. AFM was used to reveal their effects on the interfacial molecular organization and lateral structure of Curosurf films. Together with our previous study of a cholesterol-containing clinical surfactant preparation (Infasurf) (21), the present study provides novel biophysical insights into the clinical application of inhaled/instilled corticosteroids and possible untoward effects on pulmonary physiology.

\section{RESULTS}

\section{Comparison of Compression Isotherms}

Figures $\mathbf{2} \mathbf{a}$ and $\mathbf{2 b}$ compare the typical compression isotherms of pure Curosurf and Curosurf mixed with $0.1 \%, 1 \%$, and $10 \%$ cholesterol or budesonide. For all isotherms, surface pressure $(\pi)$ increased, corresponding to surface-tension decrease, when the film was compressed (i.e., reducing surface area). All compression isotherms feature a plateau region at $\pi 40-50 \mathrm{mN} / \mathrm{m}$, corresponding to a monolayer-to-multilayer transition (22).
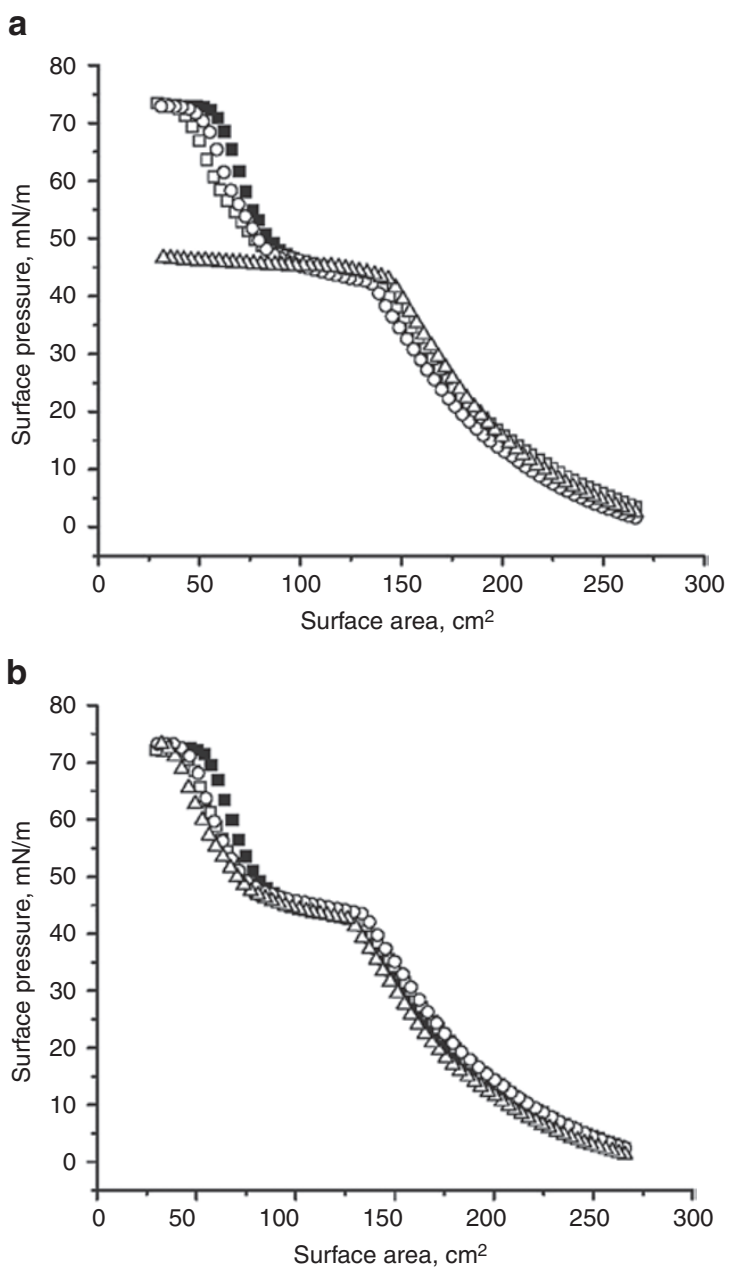

Figure 2. Effect of (a) cholesterol and (b) budesonide at various concentrations- $0.1 \%$ (open square), $1.0 \%$ (open circle), and 10\% (open triangle) of Curosurf phospholipids-on the compression isotherms of Curosurf (filled square). All preparations were spread as monolayers to an initial surface pressure $(\pi)$ of $1-3 \mathrm{mN} / \mathrm{m}$ prior to compression. Surfactant films were compressed at an identical rate of $20 \mathrm{~cm}^{2} / \mathrm{min}$ until film collapse.

As shown in Figure 2a, although the addition of $0.1 \%$ and $1 \%$ cholesterol does not significantly alter the compression isotherm of Curosurf, $10 \%$ cholesterol completely inhibits surface activity of Curosurf. With $10 \%$ cholesterol, the Curosurf film cannot be compressed to a $\pi$ higher than its equilibrium spreading pressure $\left(\pi_{\mathrm{eq}}\right)$ of $\sim 45 \mathrm{mN} / \mathrm{m}$.

By contrast, budesonide (Figure $2 \mathrm{~b}$ ), even at $10 \%$, does not significantly affect the compression isotherms of Curosurf. This comparison clearly shows the different effects of cholesterol and budesonide on surface activity of Curosurf.

\section{Comparison of Film Structures}

AFM images shown in Figure 3 compare the molecular organization and lateral structure of Curosurf with and without cholesterol at increasing $\pi$. Different cholesterol concentrations $(0,0.1 \%$, $1 \%$, and $10 \%$ to Curosurf) are compared in columns. Various $\pi$ $(20,30,40,50$, and $60 \mathrm{mN} / \mathrm{m})$ of each preparation are compared in rows. These characteristic $\pi$ were chosen to encompass the 

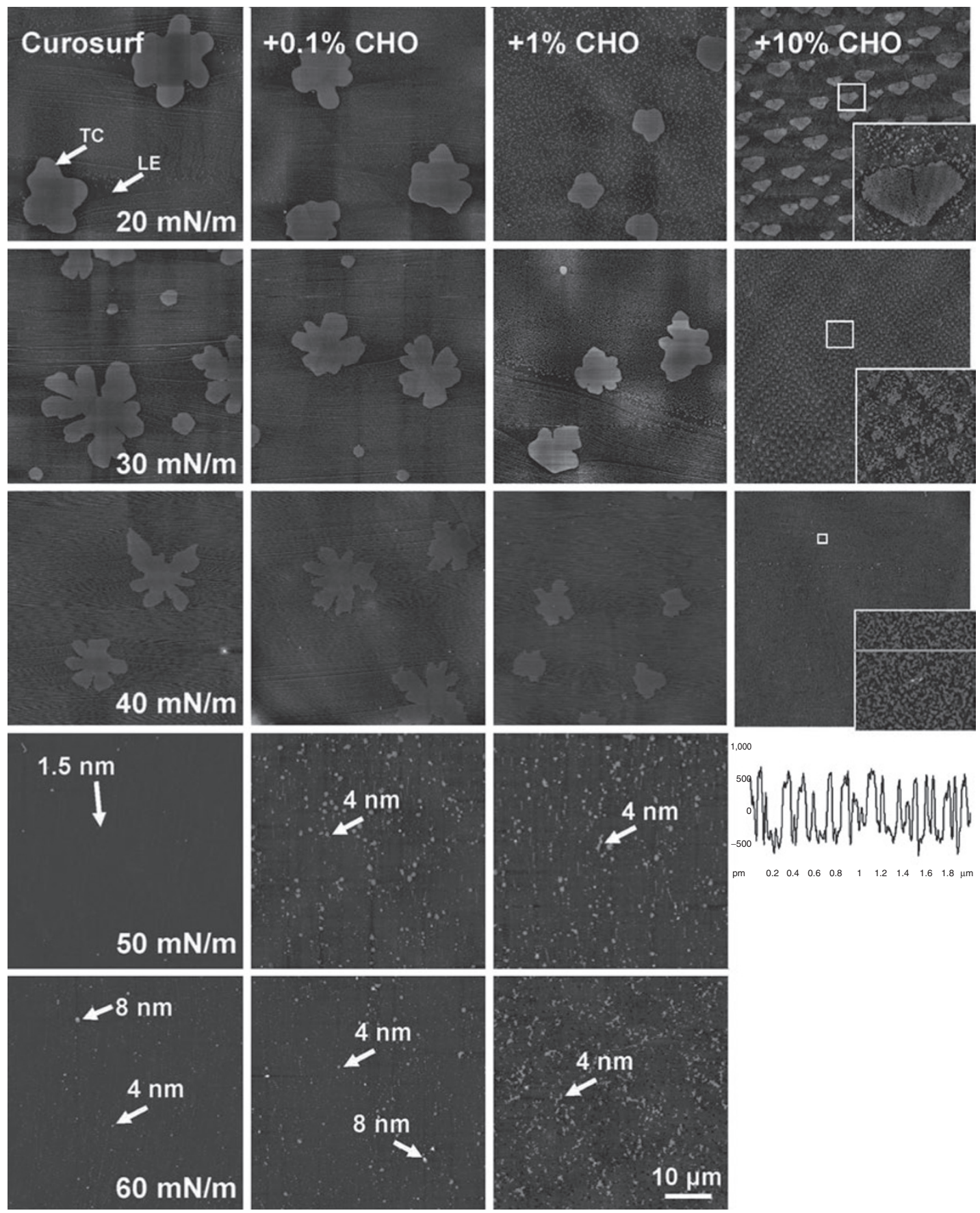

Figure 3. Effects of cholesterol $(\mathrm{CHO})$ at various concentrations on the molecular organization and lateral structure of Curosurf films. The first column shows typical AFM topographic images of pure Curosurf at five characteristic surface pressures ( $\pi$ ): 20, 30, 40, 50, and $60 \mathrm{mN} / \mathrm{m}$. Columns 2-4 show AFM topographical images of Curosurf mixed with $0.1 \%, 1.0 \%$, and $10 \% \mathrm{CHO}$, respectively. Curosurf $+\mathrm{CHO}$ films were compared with pure Curosurf at identical $\pi$ in each row. Surface pressures of 50 and $60 \mathrm{mN} / \mathrm{m}$ cannot be reached with the addition of $10 \%$ CHO due to early film collapse. The AFM scan area was $50 \times 50 \mu \mathrm{m}$ for all images (the bar equal to $10 \mu \mathrm{m}$ ). The full $z$-range was set to be $5 \mathrm{~nm}$ for monolayers (i.e., $\pi \leq 40 \mathrm{mN} / \mathrm{m}$ ) and $20 \mathrm{~nm}$ for multilayers (i.e., $\pi>40 \mathrm{mN} / \mathrm{m}$ ). Two phospholipid domains, i.e., the tilted-condensed phase (TC) and the liquid-expanded (LE) phase, are indicated by arrows.

Characteristic relative heights of multilayers are pointed by arrows. High-resolution images $(5 \times 5 \mu \mathrm{m}$ at 20 and $30 \mathrm{mN} / \mathrm{m}$, and $2 \times 2 \mu \mathrm{m}$ at $40 \mathrm{mN} / \mathrm{m})$ of critical lateral structures are indicated by rectangular boxes and shown as inserts. The bottom-right corner shows the height profile along the line tracing indicated in the insert at $40 \mathrm{mN} / \mathrm{m}$. AFM, atomic force microscopy.

complete and detailed evolution of each film under compression. Curosurf with $10 \%$ cholesterol failed to reach $\pi$ of 50 and $60 \mathrm{mN} / \mathrm{m}$. All AFM images have the same scan area of $50 \times 50 \mu \mathrm{m}$. The full $z$-range is $5 \mathrm{~nm}$ for all monolayers (i.e., $\pi \leq 40 \mathrm{mN} / \mathrm{m}$ ) and $20 \mathrm{~nm}$ for all multilayers $(\pi>40 \mathrm{mN} / \mathrm{m})$.
The first column of Figure 3 shows the lateral structure of pure Curosurf at increasing $\pi$ (22). Detailed description of these lateral structures and interpretation with respect to chemical composition and surface pressure can be found elsewhere (22). Briefly, at $\pi \leq 40 \mathrm{mN} / \mathrm{m}$, i.e., below the plateau 
region of the compression isotherm, Curosurf assumes a monolayer configuration. Two phospholipid phases-the liquid-expanded (LE) and tilted-condensed (TC) phases-are detected in the Curosurf monolayer. As labeled in Figure 3, coexistence of these phospholipid phases in the Curosurf monolayer is indicated by formation of ramified lipid domains of different relative heights, as a result of variations of lipid chain order in these phases. Lateral chemical analysis using time of flight-secondary ion mass spectroscopy (ToF-SIMS) has confirmed that the TC domains consist of disaturated phospholipids (mainly dipalmitoyl phosphatidylcholine), which extend approximately $1 \mathrm{~nm}$ higher than the surrounding LE phase that contains mainly unsaturated phospholipids and proteins (23). At $\pi$ of $50 \mathrm{mN} / \mathrm{m}$, i.e., above the plateau region of the compression isotherms, very few isolated collapse patterns of only $\sim 1.5 \mathrm{~nm}$ high appear. At $\pi$ of $60 \mathrm{mN} / \mathrm{m}$, multiple collapse patterns of $\sim 4 \mathrm{~nm}$ high appear, with very few at $\sim 8 \mathrm{~nm}$. Taking the thickness of phospholipid bilayers to be $3-5 \mathrm{~nm}$, these collapse patterns may represent multilayers one or two bilayers thick (24).

The second to fourth columns of Figure 3 show the lateral structure of Curosurf with the addition of $0.1 \%, 1 \%$, and $10 \%$ cholesterol, respectively. Compared with pure Curosurf (the first column), as cholesterol concentration rises from $0.1 \%$ to $1 \%$, more and smaller, less ramified domains form in the Curosurf monolayer (i.e., at $\pi \leq 40 \mathrm{mN} / \mathrm{m}$ ). Cholesterol in this concentration range also induces significantly more multilayers (mainly at about one bilayer thickness of $\sim 4 \mathrm{~nm}$ ) at 50 and $60 \mathrm{mN} / \mathrm{m}$. However, it should be noted that although more multilayers appear with the addition of cholesterol up to $1 \%$, these multilayers are not significantly higher than those of pure Curosurf at $60 \mathrm{mN} / \mathrm{m}$, and, more importantly, these multilayers maintain an uniform matrix structure throughout the film and thus do not appreciably inhibit surface activity of the Curosurf films.

However, when cholesterol concentration is increased to $10 \%$, the lateral structure of the Curosurf monolayer is completely changed. Instead of a few large TC domains, many much smaller domains appear at $20 \mathrm{mN} / \mathrm{m}$. Close examination of this new domain structure, as shown in the high-resolution insert $(5 \times 5 \mu \mathrm{m})$, revealed that the domains consist of loosely packed materials, in contrast to the tightly packed TC domains in pure Curosurf. A similar domain structure was found in another clinical surfactant preparation, Infasurf, which contains $5-8 \%$ cholesterol (22). This structure is probably the result of selective cholesterol intercalation into the saturated phospholipid domains, which induces a new fluid phase, i.e., the so-called liquid-ordered (LO) phase (25). Further increasing $\pi$ to 30 and $40 \mathrm{mN} / \mathrm{m}$ induces even more loosely organized structures (see the figure's inserts for detail). The bottom-right corner of Figure 3 shows the height profile along the line tracing indicated in the insert at $40 \mathrm{mN} / \mathrm{m}$. The height of these loosely packed structures varies from 0.5 to $1 \mathrm{~nm}$. Based only on the height measurement, no conclusion on the molecular organization of these structures can be drawn. However, a previous ToF-SIMS study of another clinical surfactant (BLES) with the addition of $10 \%$ cholesterol demonstrated that these new domain structures were highly enriched in cholesterol (16). When the cholesterol-mediated LO phase is predominant, the surfactant monolayer becomes so fluid that it ultimately collapses at $\pi_{\text {eq }}$.

Figure 4 shows the effect of $0.1 \%, 1 \%$, and $10 \%$ budesonide on the lateral structure of Curosurf. For ease of comparison, lateral structures of pure Curosurf at controlled $\pi$ are shown again in the first column. Several points are made in this figure. First, unlike that of cholesterol, the effect of budesonide, up to a concentration of $10 \%$, on the lateral structure the Curosurf monolayer is limited. Nevertheless, increasing budesonide concentration also seems to induce more and smaller, less ramified TC domains at the Curosurf monolayer. Second, similar to the effect of cholesterol at low concentrations (0.1 and $1 \%)$, addition of budesonide at all three concentrations appears to enhance multilayer formation at high $\pi$. This is especially evident with $10 \%$ budesonide, which failed the Langmuir-Blodgett (LB) transfer at $60 \mathrm{mN} / \mathrm{m}$. Although the film can reach high $\pi$ during compression (Figure $2 \mathbf{b}$ ), it cannot hold the high $\pi$ constant during the LB transfer. This is another indication of film fluidization, which compromises film stability. All these observations indicate that budesonide differs from cholesterol in affecting the biophysical properties of Curosurf; whereas budesonide at increasing concentration simply fluidizes the surfactant film, cholesterol induces a new phase transition.

\section{DISCUSSION}

Corticosteroids have been widely used in clinical medicine as a first-line therapy to modify the inflammatory response in many pulmonary and systemic diseases. Inhaled corticosteroids have a particular interest in that their use allows the clinician to circumvent systemic steroid side effects. However, when aerosolized corticosteroids reach the alveolar region, they must first interact with the pulmonary surfactant lining layer of the lungs. Therefore, it is vital that corticosteroids delivered via the lungs not interfere with surface activity of the surfactant lining layer.

Inhaled corticosteroid therapy has proven to be safe and effective in treating asthma in children and adults (26). However, corticosteroids administered via inhalation in preterm infants with chronic lung disease have not been shown to be efficacious. In addition, as a relatively new practice, the safety and efficacy of administering corticosteroids by intratracheal instillation in preterm infants, using exogenous surfactant as a carrier, have not been well established. It is known that preterm infants have a relatively small pool of endogenous surfactant with reduced phospholipid and protein content (27-30). Such a fragile surfactant system may be more susceptible to the introduction of foreign agents. Therefore, study of the interaction between pulmonary surfactant and corticosteroids has clinical importance as well as biophysical importance.

One rationale for the present study is that recent fundamental research has identified high cholesterol content as an important in vitro mechanism of surfactant inhibition $(16,17)$. 

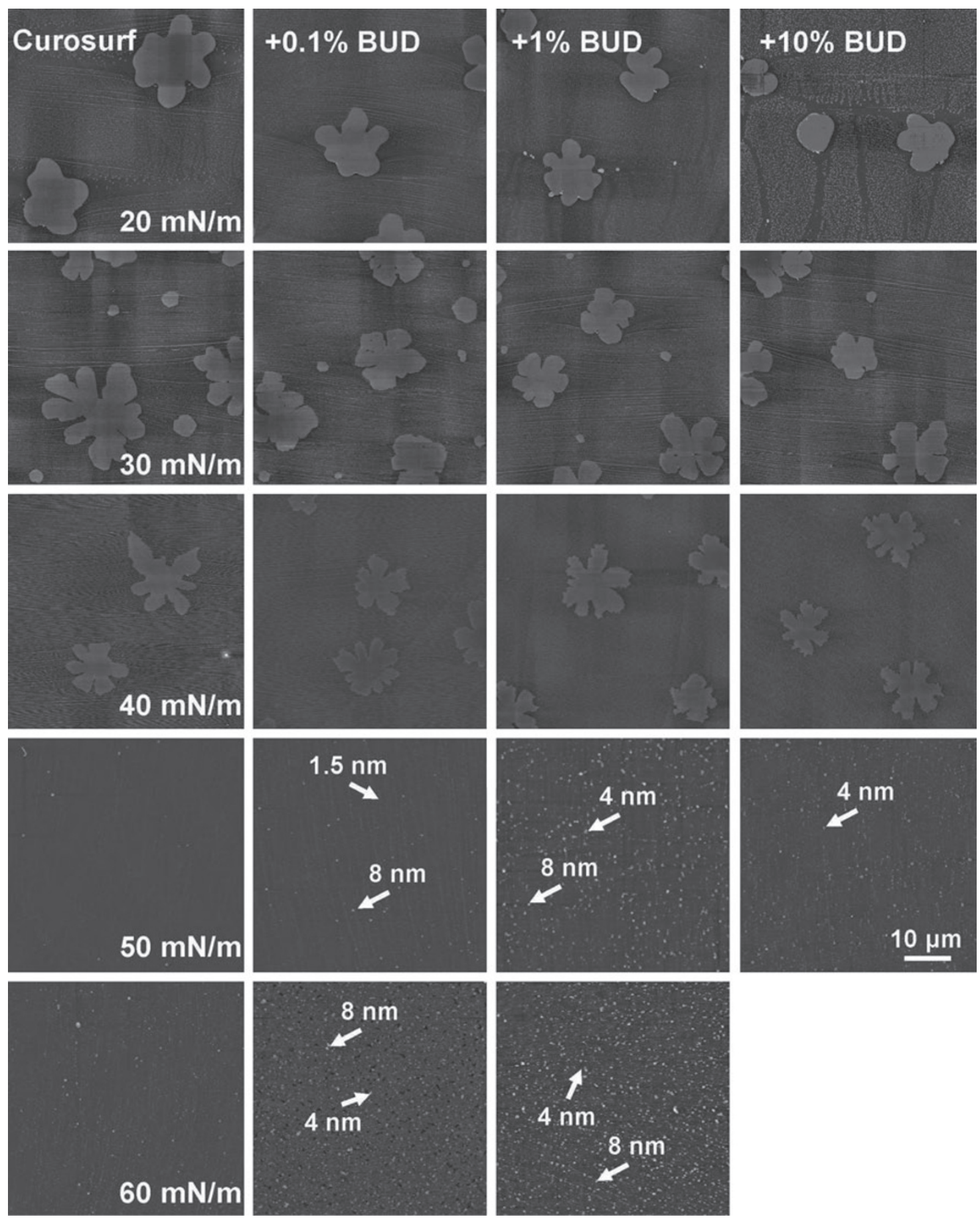

Figure 4. Effects of budesonide (BUD) at various concentrations on the molecular organization and lateral structure of Curosurf films. For ease of comparison, the first column repeats AFM topographic images of pure Curosurf at five characteristic surface pressures ( $\pi$ )—20, 30, 40, 50, and $60 \mathrm{mN} / \mathrm{m}$-as shown in the first column of Figure 3. Columns 2-4 show AFM topographic images of Curosurf mixed with $0.1 \%, 1.0 \%$, and $10 \%$ BUD, respectively.

Curosurf + BUD films were compared with pure Curosurf at identical $\pi$ in each row. Langmuir-Blodgett film transfer failed for Curosurf mixed with 10\% BUD at $60 \mathrm{mN} / \mathrm{m}$. The AFM scan area was $50 \times 50 \mu \mathrm{m}$ for all images (bar $=10 \mu \mathrm{m}$ ). The full $z$-range was set to be $5 \mathrm{~nm}$ for monolayers (i.e., $\pi \leq 40 \mathrm{mN} / \mathrm{m}$ ) and $20 \mathrm{~nm}$ for multilayers (i.e., $\pi>40 \mathrm{mN} / \mathrm{m}$ ). Characteristic relative heights of multilayers are indicated by arrows. AFM, atomic force microscopy.

Corticosteroids have the same steroid core as cholesterol (Figure 1), and when corticosteroids are instilled into the airway, their local concentration in the alveolar hypophase can be very high. Therefore, it is a relevant concern whether the effect of corticosteroids on inhibition of surface activity of pulmonary surfactant is similar to that of cholesterol.
The present study found differential effects of cholesterol and budesonide on the biophysical properties of an animal-derived surfactant preparation, Curosurf. We found that, although at low concentrations $(0.1 \%$ and $1 \%)$, neither cholesterol nor budesonide significantly affects the surface activity of Curosurf, 10\% cholesterol completely inhibits its surface-tension-lowering 
ability. By contrast, budesonide at the same concentration only moderately reduces film stability and does not significantly alter the compression isotherm.

Study of lateral film structures using AFM revealed that both cholesterol and budesonide at increasing concentration play a role in fluidizing the surfactant film, indicated by formation of less ramified domains at the Curosurf monolayer and by promotion of more multilayers at high $\pi$. Curosurf consists of $\sim 99 \%$ phospholipids and $\sim 1 \%$ proteins, with nearly no neutral lipids (22). The addition of small steroid molecules is expected to enhance the lateral diffusion of phospholipid molecules.

Nevertheless, $10 \%$ cholesterol, but not budesonide, completely alters the "solid-fluid" (i.e., TC-LE) phase coexistence at the Curosurf monolayer. Cholesterol at $10 \%$ of phospholipids in weight is equivalent to $\sim 20 \%$ in mole fraction. It is known that cholesterol in this concentration range can induce a unique LO phase that fluidizes the lipids in gel-phase bilayers and in TC-phase monolayers (25). By disturbing lipid packing in these "solid" phases, cholesterol can significantly enhance fluidity and lateral diffusion rate and at the same time prevent a lipid monolayer from becoming tightly packed beyond its equilibrium spreading pressure. This unique cholesterol-mediated phase transition contributes to surfactant inhibition.

It should be noted that endogenous surfactant contains $5-10 \%$ cholesterol by weight. In addition, previous studies based on a BLES have demonstrated that cholesterol-induced surfactant inhibition becomes significant only with the addition of at least $20 \mathrm{wt} \%$ cholesterol $(16,17)$. However, the present study showed that cholesterol at $10 \mathrm{wt} \%$ has a significant inhibition effect on Curosurf. The reason for this discrepancy is currently unclear, but it may be related to the particular phospholipid profiles of this porcine surfactant.

The differential effects of cholesterol and budesonide on the biophysical properties of pulmonary surfactant are most likely related to the alkyl side chain at C-17 of the cholesterol molecule (see Figure 1a) (14). The 8-10-carbon alkyl side chain at C-17 position, which is unique to cholesterol, is known to be essential for the cholesterol molecule to exert its characteristic effects on both model and biological membranes $(31,32)$.

In contrast to cholesterol, budesonide plays a simple role of film fluidization without inducing new phase transitions. Budesonide is one of the most commonly used corticosteroids for inhalation therapy and has been clinically tested with intratracheal administration to treat premature newborns with or at high risk for chronic lung disease (8). To date, the dose of inhaled/instilled budesonide used in clinical practice has not been optimized, but it is commonly used at a very low concentration-less than $0.5 \%$ of pulmonary surfactant. (For inhaled steroids, the dose reaching the lungs can be much lower because the aerosol-delivery efficiency for ventilated infants is significantly lower than that for spontaneously breathing or ventilated adults (33).) This low dose of corticosteroids is in part due to concern regarding potential impairment of the surface activity of pulmonary surfactant $(8,18,19)$. Our present study suggests that a higher concentration of budesonide might be clinically feasible without significant adverse effects on the surface activity. Confirmation of this will require further investigation with animal models.

It is important to note that, for intratracheal administration of corticosteroids using clinical surfactant as a delivery vehicle, optimal performance may depend on the selection of both the surfactant preparation and the steroid drug, as well as their mixing ratio. In a separate study, we showed that $10 \%$ budesonide, although not appreciably affecting surface activity of Curosurf as shown in the present study, significantly decreased surface activity of another clinical surfactant preparation, Infasurf (21). A main compositional difference between Infasurf and Curosurf is that Infasurf contains $5-8 \%$ cholesterol whereas Curosurf is cholesterol-free (22). Our finding is qualitatively consistent with the observations by Palmer et al., who found that $0.6 \%$ budesonide increased the minimum surface tension of a cholesterol-containing surfactant preparation (BLES with 2-3\% cholesterol (ref. 22)) but had no deleterious effect on a cholesterol-free surfactant preparation (Survanta) (18). Yeh et al. also showed that $2 \%$ budesonide added to Survanta did not elevate its minimum surface tension (8). Although the phospholipid and protein profiles of these clinical preparations also differ slightly (22), these studies suggest that cholesterol and budesonide together decrease the surface activity of clinical surfactants. Our previous mechanism study further suggested that a specific hydrophobic interaction between cholesterol and budesonide may be responsible for this deleterious effect (21). Therefore, our studies suggest that cholesterol-free surfactant preparations, such as Curosurf (used in animal models) $(9,11)$ and Survanta (used in clinical trials) $(8,10)$, may be more suitable than cholesterol-containing surfactant preparations (such as Infasurf and BLES) as a carrier of budesonide for intratracheal administration.

In conclusion, we have demonstrated differential effects of cholesterol and budesonide on the biophysical properties of Curosurf. At a low concentration (up to 1\%), both steroids play a similar role in fluidizing the surfactant film. However, when the concentration is increased to $10 \%$, cholesterol induces a unique phase transition that abolishes surface activity of the Curosurf film. By contrast, $10 \%$ budesonide simply fluidizes the film, thus having only a limited effect on surface activity. These differences are most likely related to the unique alkyl chain at the C-17 position of cholesterol. Together with a previous study using Infasurf (21), our studies suggest that cholesterol-free surfactant preparations may have an advantage over cholesterol-containing preparations as a carrier of budesonide in that a larger amount of the drug may be delivered to the lungs without significantly compromising the surface activity of pulmonary surfactant.

\section{METHODS}

\section{Materials}

Curosurf (poractant alfa) was a gift from Cornerstone Therapeutics. (Cary, NC). It is a modified natural surfactant prepared from minced porcine lung tissue. Curosurf contains $~ 99$ wt $\%$ phospholipids and $1 \%$ hydrophobic surfactant proteins (SP-B and SP-C) (22). It is devoid of all neutral lipids (including cholesterol) as a result of undergoing gel chromatography during manufacture. Curosurf was extracted with chloroform-methanol, dried under a nitrogen stream, and redissolved 
in chloroform to a final concentration of $1 \mathrm{mg}$ phospholipids per $\mathrm{ml}$. All stock solutions were stored at $-20^{\circ} \mathrm{C}$ until use. Cholesterol and budesonide were purchased from Sigma-Aldrich (St Louis, MO) and used without further purification.

\section{Sample Preparation}

Both cholesterol and budesonide were studied at a wide range of concentration ratios with respect to the phospholipids in Curosurf. For the stock solution of $1 \mathrm{mg} / \mathrm{ml}$ Curosurf, each steroid was added at concentrations of $0.001,0.01$, and $0.1 \mathrm{mg} / \mathrm{ml}$, corresponding to $0.1 \%, 1 \%$, and $10 \%$ of phospholipids in Curosurf, respectively. To date, inhaled/instilled corticosteroids tested in vitro $(18,19)$, in animal models $(9-11,19)$, and in clinical practice $(5,8)$ have commonly been in the range of $0.2-0.6 \%$ of surfactant. Therefore, although the surfactant concentration used in the present in vitro study is much lower than that used clinically, this study covers all mixing ratios between the corticosteroid and surfactant reported in the literature.

\section{Langmuir Balance}

The surface activity of Curosurf with and without steroids was evaluated by measuring surface pressure-surface area $(\pi-A)$ isotherms using a Langmuir balance (KSV Nima, Coventry, UK) at room temperature $\left(20 \pm 1^{\circ} \mathrm{C}\right)$. Surface pressure $(\pi)$ and surface tension $(\gamma)$ are linearly correlated by $\pi=\gamma_{0}-\gamma$, where, $\gamma_{0}$ is the surface tension of a clean air-water interface, approximately equal to $72 \mathrm{mN} / \mathrm{m}$ at room temperature. Thus, the increase in $\pi$ corresponds to the extent that a film decreases $\gamma$.

Details of our experimental procedures can be found elsewhere $(21,22)$. Briefly, Curosurf films with/without steroids were spread on pure water to increase $\pi$ to $1-3 \mathrm{mN} / \mathrm{m}$ and were left undisturbed for $10 \mathrm{~min}$ to allow evaporation of solvent. The spread films were compressed at a rate of $20 \mathrm{~cm}^{2} / \mathrm{min}$, with the $\pi-\mathrm{A}$ isotherms recorded. All compression isotherms were studied at least three times to ensure reproducibility.

For AFM imaging, the surfactant film at the air-water interface was transferred to a solid substrate under controlled $\pi$ using the LB technique. Specifically, surfactant films at characteristic $\pi$ of $20,30,40,50$, and $60 \mathrm{mN} / \mathrm{m}$ were deposited to freshly peeled mica surfaces at a dipping rate of $1 \mathrm{~mm} / \mathrm{min}$. If $60 \mathrm{mN} / \mathrm{m}$ could not be reached, surfactant films at the highest possible $\pi$ were studied. LB transfer of each sample at each $\pi$ was repeated at least three times to ensure reproducibility.

\section{AFM}

Topographical images of the LB samples were obtained using an Innova AFM (Bruker, Santa Barbara, CA). Samples were scanned in air at multiple locations with various scan areas to ensure detection of representative structures. Both contact and tapping modes were used. The different scan modes gave equivalent results. A silicon nitride cantilever with a spring constant of $0.12 \mathrm{~N} / \mathrm{m}$ and a nominal tip radius of $2 \mathrm{~nm}$ was used in contact mode, and a silicon probe with a resonance frequency of $300 \mathrm{kHz}$ and a spring constant of $40 \mathrm{~N} / \mathrm{m}$ was used in tapping mode. AFM images were analyzed using Nanoscope software (version 7.30).

\section{ACKNOWLEDGMENTS}

The authors thank Alan Roberts at Cornerstone Therapeutics Inc. for the generous donation of Curosurf samples and Lloyd Hihara for sharing facilities.

\section{STATEMENT OF FINANCIAL SUPPORT}

This work was supported by the Leahi Fund to Treat \& Prevent Pulmonary Disease (44936) from the Hawaii Community Foundation (Y.Y.Z.) and the RMATRIX awards (US4RR026136) from the National Institutes of Health (Y.Y.Z. and C.R.N.). H.Z. was supported by a faculty-exchange program between Peking University and the University of Hawaii at Manoa, operated by the Center for Chinese Studies.

\section{REFERENCES}

1. Jobe AH. Postnatal corticosteroids for bronchopulmonary dysplasia. Clin Perinatol 2009;36:177-88.

2. Cole CH, Fiascone JM. Strategies for prevention of neonatal chronic lung disease. Semin Perinatol 2000;24:445-62.

3. Niwas R, Kamat M, Kling P, Yeh TF, Pyati S. Postnatal corticosteroids to treat or to prevent chronic lung disease: a survey of neonatologists' practices. Arch Dis Child Fetal Neonatal Ed 2009;94:F77.
4. American Academy of Pediatrics. Committee on Fetus Newborn, Canadian Paediatric Society. Fetus and Newborn Committee. Postnatal corticosteroids to treat or prevent chronic lung disease in preterm infants. Pediatrics 2002;109:330-8.

5. Cole $\mathrm{CH}$, Colton T, Shah BL, et al. Early inhaled glucocorticoid therapy to prevent bronchopulmonary dysplasia. N Engl J Med 1999;340:1005-10.

6. Shah V, Ohlsson A, Halliday HL, Dunn MS. Early administration of inhaled corticosteroids for preventing chronic lung disease in ventilated very low birth weight preterm neonates. Cochrane Database Syst Rev 2007:CD001969.

7. Shah SS, Ohlsson A, Halliday H, Shah VS. Inhaled versus systemic corticosteroids for the treatment of chronic lung disease in ventilated very low birth weight preterm infants. Cochrane Database Syst Rev 2007:CD002057.

8. Yeh TF, Lin HC, Chang CH, et al. Early intratracheal instillation of budesonide using surfactant as a vehicle to prevent chronic lung disease in preterm infants: a pilot study. Pediatrics 2008;121:e1310-8.

9. Dani C, Corsini I, Burchielli S, et al. Natural surfactant combined with beclomethasone decreases oxidative lung injury in the preterm lamb. Pediatr Pulmonol 2009;44:1159-67.

10. Yang CF, Jeng MJ, Soong WJ, Lee YS, Tsao PC, Tang RB. Acute pathophysiological effects of intratracheal instillation of budesonide and exogenous surfactant in a neonatal surfactant-depleted piglet model. Pediatr Neonatol 2010;51:219-26.

11. Dani C, Corsini I, Burchielli S, et al. Natural surfactant combined with beclomethasone decreases lung inflammation in the preterm lamb. Respiration 2011;82:369-76.

12. Zuo YY, Veldhuizen RA, Neumann AW, Petersen NO, Possmayer F. Current perspectives in pulmonary surfactant-inhibition, enhancement and evaluation. Biochim Biophys Acta 2008;1778:1947-77.

13. Veldhuizen R, Nag K, Orgeig S, Possmayer F. The role of lipids in pulmonary surfactant. Biochim Biophys Acta 1998;1408:90-108.

14. Ghosh AK, Pore N, Basu R, De S, Nandy P. Lipid perturbation by corticosteroids: An anisotropic study. Colloids Surf B Biointerfaces 1996;7:65-8.

15. Bernardino de la Serna J, Perez-Gil J, Simonsen AC, Bagatolli LA. Cholesterol rules: direct observation of the coexistence of two fluid phases in native pulmonary surfactant membranes at physiological temperatures. J Biol Chem 2004;279:40715-22.

16. Keating E, Rahman L, Francis J, et al. Effect of cholesterol on the biophysi$\mathrm{cal}$ and physiological properties of a clinical pulmonary surfactant. Biophys J 2007;93:1391-401.

17. Gunasekara L, Schürch S, Schoel WM, et al. Pulmonary surfactant function is abolished by an elevated proportion of cholesterol. Biochim Biophys Acta 2005; 1737:27-35.

18. Palmer D, Schürch S, Belik J. Effect of budesonide and salbutamol on surfactant properties. J Appl Physiol 2000;89:884-90.

19. Nimmo AJ, Carstairs JR, Patole SK, Whitehall J, Davidson K, Vink R. Intratracheal administration of glucocorticoids using surfactant as a vehicle. Clin Exp Pharmacol Physiol 2002;29:661-5.

20. Davies MJ, Brindley A, Chen X, Doughty SW, Marlow M, Roberts CJ. A quantitative assessment of inhaled drug particle-pulmonary surfactant interaction by atomic force microscopy. Colloids Surf B Biointerfaces 2009;73:97-102

21. Wang YE, Zhang H, Fan Q, Neal CR, Zuo YY. Biophysical interaction between corticosteroids and natural surfactant preparation: implications to pulmonary drug delivery using surfactant as a carrier. Soft Matter 2012;8:504-11.

22. Zhang H, Fan Q, Wang YE, Neal CR, Zuo YY. Comparative study of clinical pulmonary surfactants using atomic force microscopy. Biochim Biophys Acta 2011;1808:1832-42.

23. Keating E, Waring AJ, Walther FJ, Possmayer F, Veldhuizen RA, Petersen NO. A ToF-SIMS study of the lateral organization of lipids and proteins in pulmonary surfactant systems. Biochim Biophys Acta 2011;1808: 614-21.

24. Zhang H, Wang YE, Fan Q, Zuo YY. On the low surface tension of lung surfactant. Langmuir 2011;27:8351-8. 
25. Mouritsen OG, Zuckermann MJ. What's so special about cholesterol? Lipids 2004;39:1101-13.

26. Adams N, Lasserson TJ, Cates CJ, Jones PW. Fluticasone versus beclomethasone or budesonide for chronic asthma in adults and children. Cochrane Database Syst Rev 2007:CD002310.

27. Zuo YY, Alolabi H, Shafiei A, et al. Chitosan enhances the in vitro surface activity of dilute lung surfactant preparations and resists albumin-induced inactivation. Pediatr Res 2006;60:125-30.

28. Ballard PL, Merrill JD, Godinez RI, Godinez MH, Truog WE, Ballard RA. Surfactant protein profile of pulmonary surfactant in premature infants. Am J Respir Crit Care Med 2003;168:1123-8.

29. Merrill JD, Ballard RA. Pulmonary surfactant for neonatal respiratory disorders. Curr Opin Pediatr 2003;15:149-54.
30. Merrill JD, Ballard RA, Cnaan A, et al. Dysfunction of pulmonary surfactant in chronically ventilated premature infants. Pediatr Res 2004;56: 918-26.

31. Vilchèze $\mathrm{C}$, McMullen TP, McElhaney RN, Bittman R. The effect of sidechain analogues of cholesterol on the thermotropic phase behavior of 1-stearoyl-2-oleoylphosphatidylcholine bilayers: a differential scanning calorimetric study. Biochim Biophys Acta 1996;1279:235-42.

32. McMullen TP, Vilchèze C, McElhaney RN, Bittman R. Differential scanning calorimetric study of the effect of sterol side chain length and structure on dipalmitoylphosphatidylcholine thermotropic phase behavior. Biophys J 1995;69:169-76.

33. Cole $\mathrm{CH}$. Special problems in aerosol delivery: neonatal and pediatric considerations. Respir Care 2000;45:646-51. 